\title{
Low field strength magnetic resonance imaging of the neonatal brain
}

\author{
E H Whitby, M N Paley, M F Smith, A Sprigg, N Woodhouse, P D Griffiths
}

Arch Dis Child Fetal Neonatal Ed 2003;88:F203-F208

See end of article for authors' affiliations

...................

Correspondence to: Dr Whitby, Department of Academic Radiology, Floor C, Royal Hallamshire Hospital, Glossop Road, Sheffield S10 2JF, UK; e.whitby@sheffield.ac.uk

Accepted 5 August 2002
Background: Magnetic resonance (MR) imaging of the neonate has been restricted by the need to transport the sick baby to the large magnetic resonance scanners and often the need for sedation or anaesthesia in order to obtain good quality images. Ultrasound is the reference standard for neonatal imaging.

Objective: To establish a dedicated neonatal MR system and compare the clinical usefulness of MR imaging with ultrasound imaging.

Design: Prospective double blind trial.

Setting: Neonatal intensive care unit, Sheffield.

Main outcome measures: Imaging reports.

Patients: 134 premature and term babies.

Results: In $56 \%$ of infants with pathology suspected on clinical grounds, MR provided additional useful clinical information over and above that obtained with ultrasound.

Conclusion: Infants can be safely imaged by dedicated low field magnetic resonance on the neonatal intensive care unit without the need for sedation at a cost equivalent to ultrasound.
$\mathrm{T}$ he brain of the fetus and neonate is vulnerable to a variety of insults. Premature infants are at greatest risk, when the combination of prenatal, intrapartum, and postnatal factors produce a high risk of brain damage from haemorrhagic and/or hypoxic/ischaemic lesions. ${ }^{1}$ As $6 \%$ of babies in the United Kingdom are born preterm (unpublished data from the special care baby unit of Jessop Hospital), this presents a serious health challenge. The sequelae of brain injury in the neonatal period include motor, cognitive, language, and learning defects, which are a major cause of morbidity and mortality throughout childhood. ${ }^{1}$

Detection of haemorrhagic complications in the newborn infant by ultrasound has been well described.$^{2}{ }^{3}$ It is accepted that it cannot reliably identify ischaemic changes in white matter, which may result in later learning difficulties. ${ }^{34}$ Experience with magnetic resonance (MR) imaging in older children and adults has shown that it is sensitive in detecting hypoxic/ischaemic damage ${ }^{5}$ and therefore would also be expected to be good for detecting this type of pathology in the newborn. Studies using high field strength scanners have shown that this is the case. ${ }^{6-9}$ However, MR of sick babies cared for on neonatal intensive care units is extremely difficult because MR machines are typically in departments elsewhere in the hospital. They are not designed for small babies, who require close monitoring and often sedation for the procedure. Ideally, imaging should take place on the unit, in a clinical environment designed to support the infant.'

In this report, we describe our initial experience of siting a small, low field strength $(0.2 \mathrm{~T})$ magnetic resonance scanner on our neonatal intensive care unit and comparing the results with those of ultrasound.

\section{PATIENTS AND METHODS}

\section{The infants}

Ethical approval for the study was given by the South Sheffield ethics committee. The babies were all on the neonatal intensive care unit at the Jessop Hospital, and parental consent was obtained in all cases. A total of 134 babies were included.
Group 1 consisted of infants with no neurological symptoms and not expected to have intracranial disease. Group 2 patients had known or suspected intracranial pathology on clinical grounds. Before the MR examination, the babies were swaddled in a blanket and placed in a perspex "pod". All medical treatment was maintained during scanning. No sedation or anaesthesia was required.

\section{The MR system}

A specialised MR system (InnerVision MRI Ltd, London, UK) operating at $0.17 \mathrm{~T}(7.2 \mathrm{MHz})$ and using $15 \mathrm{mT} / \mathrm{m}$ gradients was installed in a modular screened enclosure $(1.5 \times 1 \mathrm{~m})$ within a small room $(4 \times 2.5 \mathrm{~m})$ adjacent to the neonatal intensive care unit (fig 1 ).

The system was passively shimmed to achieve a $16 \mathrm{~cm}$ uniform field ( 5 ppm full width half maximum (FWHM)).

A low magnet weight of $500 \mathrm{~kg}$ and compact size $(1.1 \times 0.5$ $\times 0.5 \mathrm{~m}$ ) meant that the system could be installed on the fourth floor of the hospital using a regular goods lift without floor reinforcing. MR compatible pulse oximetry (MR 3500; MR Resources Inc, Florida, USA) was used to assess blood oxygen saturation and pulse rate through an infrared probe. All monitoring equipment leads were fed through waveguides into the enclosure.

\section{MR sequences}

Images were acquired using either a $15 \mathrm{~cm}$ diameter loop coil or $15 \mathrm{~cm}$ diameter volume coil in linear transit receive mode using a $100 \mathrm{~W}$ radiofrequency amplifier. The fringe field of the magnet $(300 \mathrm{~mm})$ was completely enclosed within the screened enclosure. T1 and T2 weighted spin echo, gradient echo, and inversion recovery sequences were acquired. A typical examination included axial and coronal $\mathrm{Tl}$ weighted scans $(\mathrm{TR} / \mathrm{TE} 600 / 20$; slice thickness $=5 \mathrm{~mm} ; \mathrm{FOV}=160 \mathrm{~mm}$; matrix $=160 \times 256 ; \mathrm{NEX}=3$; acquisition time 4.8 minutes $)$ and a coronal T2 weighted sequence (TR/TE 3000/120; slice thickness $=5 \mathrm{~mm}$; FOV $=160 \mathrm{~mm}$; matrix $=128 \times 256$; NEX $=1$; acquisition time 6.4 minutes). Image positions were planned from a set of axial, sagittal, and coronal scouts run as 


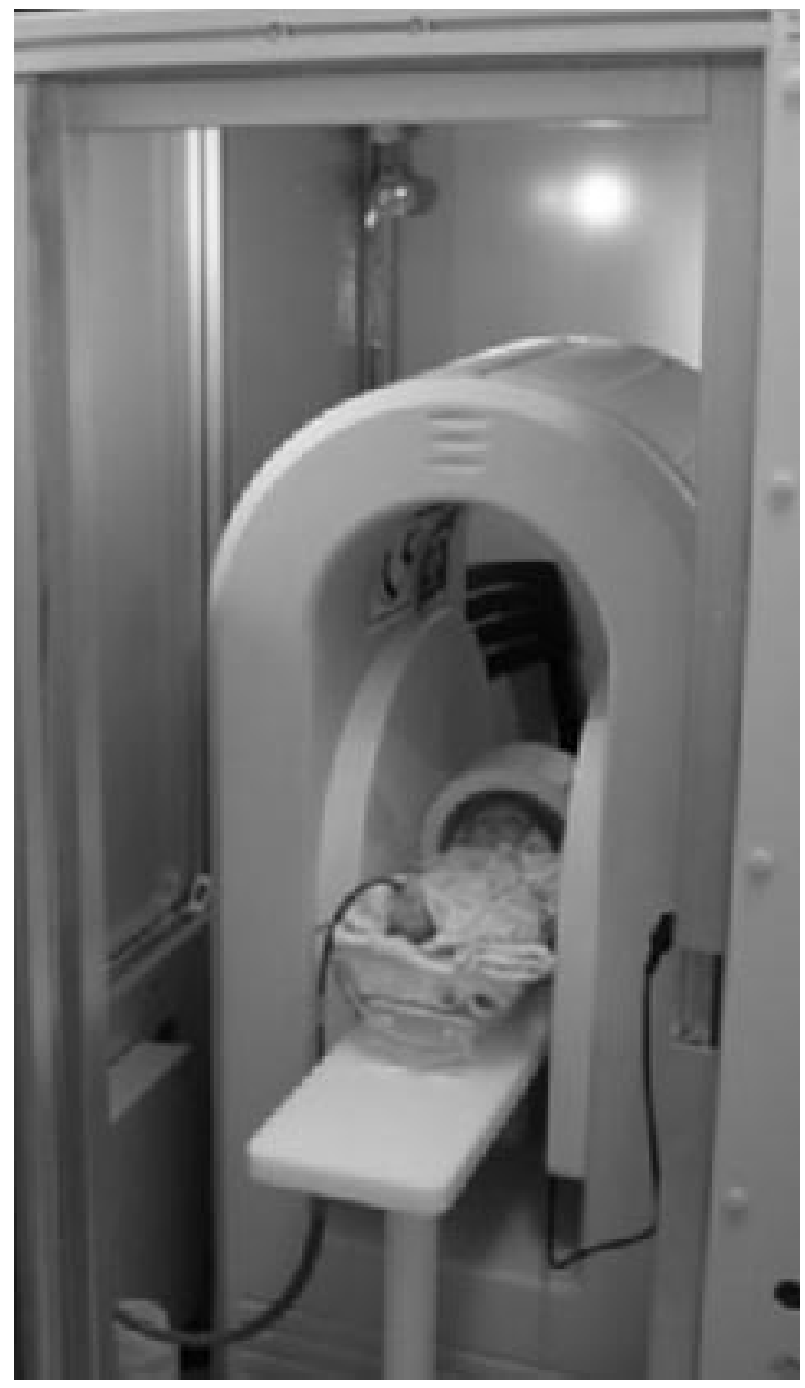

Figure 1 The magnetic resonance scanner.

sequential fast acquisitions. Centre frequency and radiofrequency pulse amplitude were calibrated automatically before each scan. Specific absorption rate was calibrated for each study and was typically a factor of 10 lower than the National Radiation Protection Board guidelines. ${ }^{10}$ The scanner was very quiet: the measured noise level for the $\mathrm{Tl}$ weighted sequence was less than $65 \mathrm{~dB}$.

Images were transferred over a network to a review station using Dicom compliant software (PiView 2.0; Mediface, Seoul, Korea) and archived to Zip disk. In selected cases, images were printed to a Dicom compliant laser camera (Dryview 8700; Kodak, Rochester, New York, USA).

Figure 2 shows a normal Tl weighted coronal scan in a term infant.

\section{Ultrasound technique}

All the scans were performed on the neonatal intensive care unit using a Toshiba 140 scan machine with 5 or $7 \mathrm{MHz}$ electronic sector probes via the anterior and lateral fontanelles. Imaging was performed using standard coronal, sagittal, parasagittal, and axial imaging planes. The scans were performed in real time and reported by the consultant paediatric radiologist unaware of the MR findings. Ultrasound and MR imaging were performed within 24 hours of each other. Standard image sections were recorded on thermal paper prints. The ultrasound reports were the clinical reports and not retrospective reviews of the ultrasound images.

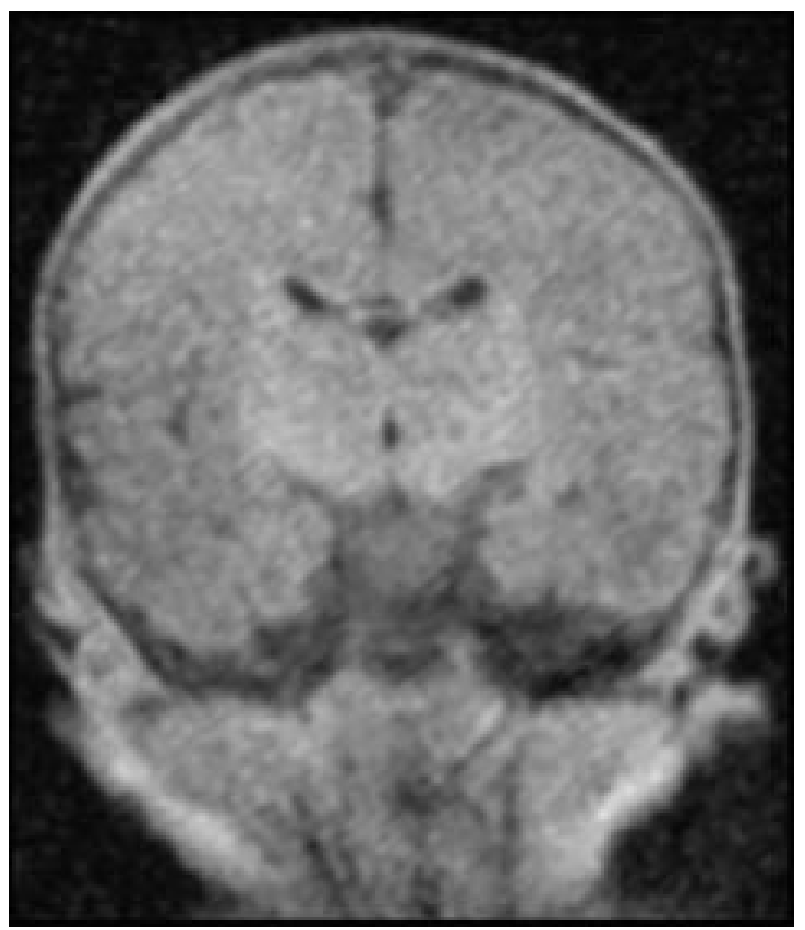

Figure $2 \mathrm{Tl}$ weighted coronal scan of the brain of a normal term baby.

\section{Image reporting and analysis}

Images were independently reviewed for pathology by two experienced radiologists (EW, PDG) who were blinded to the ultrasound results. Results are expressed according to whether they agreed with the ultrasound findings and whether this altered clinical management of the infant. A $2 \times 2$ contingency table was formed for detection of pathology by the two imaging modalities. A $\chi^{2}$ test was performed with the Yates continuity correction, and significance attributed at $\mathrm{p}<0.001$.

\section{RESULTS}

\section{Group 1: control cases}

The purpose of scanning controls was to optimise the scan parameters before attempting to handle sick neonates. This was a novel application for this MR system so the scan parameters could not be inferred from other protocols. All had axial and coronal $\mathrm{Tl}$ weighted and coronal $\mathrm{T} 2$ weighted scans, using a range of TR and TE values. All 89 control neonates were neurologically intact on clinical assessment at the time of scanning and all but one have developed normally during follow up (range 2-21 months) as assessed clinically by a neonatologist. The controls included 40 preterm and 49 term infants. Movement artefacts occurred in three images (3.4\%) making them difficult to interpret. All examinations were normal except for one child with an established left middle cerebral artery territory infarct (fig 3), which was not clearly visible on cranial ultrasound. This was thought to have resulted from an in utero event in the third trimester.

\section{Group 2: infants with suspected pathology}

There were 43 neonates ( 20 term deliveries, 23 premature) in this group. The median birth weight of the group was $1415 \mathrm{~g}$ (range 670-4110), and the median weight at the time of the scan was $2000 \mathrm{~g}$ (range 880-4900). The mean age of the babies at the time of the scan was 16.2 days (range 5 hours to 332 days). The median gestational age at the time of delivery was 30 weeks (range 24 weeks-term). 


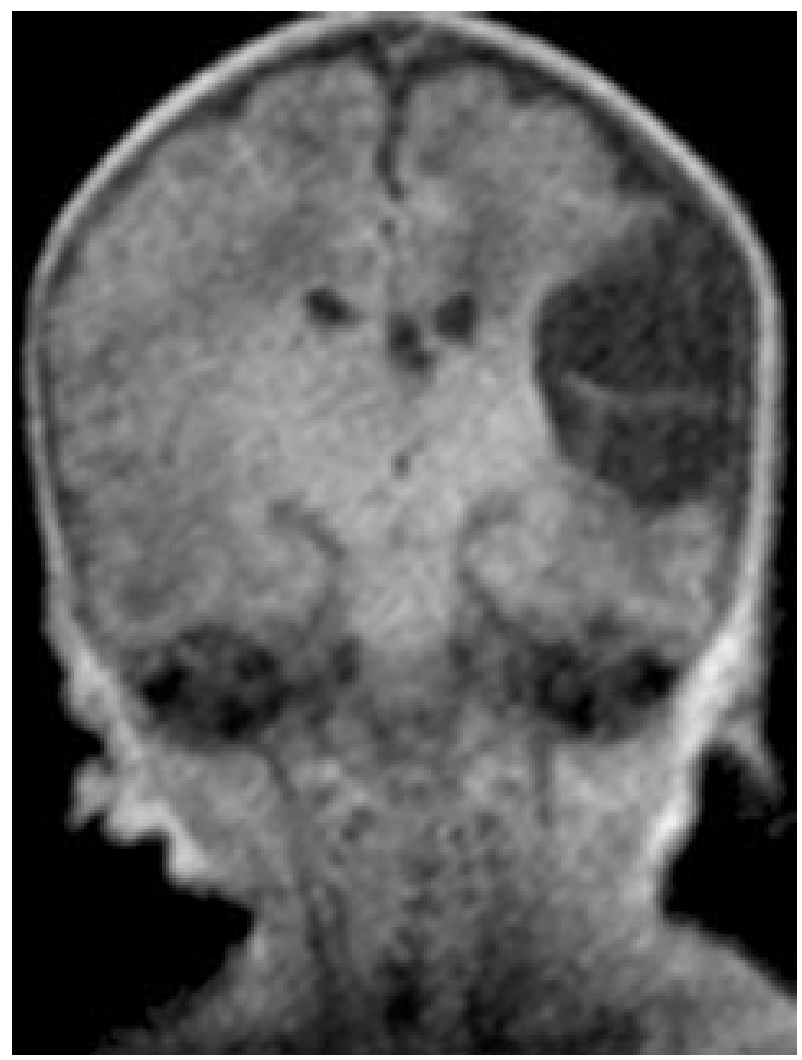

Figure 3 Coronal T1 weighted scan showing an established middle cerebral artery territory infarct.

Table 1 Abnormal cases where the information from the ultrasound and magnetic resonance (MR) scans was diagnostic and equivalent

\begin{tabular}{ll}
\hline Diagnosis & Number of patients \\
\hline Congenital malformations & 5 \\
Dandy Walker syndrome & 1 \\
Frontal encephalocele & 1 \\
Hydrocephalus and spina bifida & 2 \\
Intraventricular neuroepithelial cyst & 1 \\
Periventricular leucomalacia* (cystic) & 4 \\
Middle cerebral artery territory infarctions* & 2 \\
Hydrocephalus and intraventricular & 1 \\
haemorrhage & \\
Bright thalami on ultrasound and MR (suggests & 1 \\
hypoxic injury) & \\
Abnormal signal in the left temporal lobe of & 1 \\
uncertain nature &
\end{tabular}

*Although the diagnosis was made on both ultrasound and MR, the MR scans showed the lesions more clearly and the extent of the lesion was easily displayed by the MR scans.

All MR examinations in this group were reportable $(0 \%$ failure rate). All 43 scans were compared with ultrasound findings. In no case was the brain normal on both modalities. In $14 / 43(33 \%)$ cases, the brain was abnormal on both modalities and gave equivalent information (table 1). In 24/43 (56\%) cases, MR gave more information than ultrasound, including 17 where ultrasound was normal (table 2). In 5/43 $(12 \%)$, ultrasound showed grade one germinal matrix haemorrhages not seen on MR.

The $\chi^{2}$ test for MR detecting more pathology than ultrasound was highly significant $\left(p<0.001, \chi^{2}=16.86\right.$, $\mathrm{df}=1)$.

Typical time in the scanner was 30-40 minutes. The time for each sequence of scans to be obtained was $4.5-6$ minutes. The neonates all tolerated the scans. Only three of the scans could not be interpreted because of movement artefact, and one of these was successfully repeated the following day. All of the babies had an ultrasound scan of their brains within 24 hours of the MR scan.

\section{DISCUSSION}

Ultrasound is the most commonly used cranial imaging modality in the newborn. It is easy to perform by trained personnel, causes minimal disruption to the baby, and can be performed on even very ill babies in incubators and on ventilators. However, it is operator, machine, and probe dependent and limited by fontanelle size, depth of penetration, and the angulated view required. This results in poor views of the periphery of the brain and posterior fossa. It is poor at differentiating grey from white matter. Its limited accuracy in imaging the neonatal brain is evidenced by studies suggesting large interobserver variation. ${ }^{9}$

MR does not use ionising radiation. It has the important advantage of multiplanar imaging, which makes the detection of most pathology much easier in areas in which ultrasound and computed tomography are inefficient, such as in the posterior fossa, adjacent to the tentorium, and under the occipital and temporal lobes. MR has been shown to detect all stages of intraparenchymal lesions described pathophysiologicallythat is, haemorrhages, cystic changes leading to resolution with or without atrophy, and ventricular dilatation. Most MR studies use high field strength systems. ${ }^{6-8}$ These have been shown to cause fluctuations in the vital signs of the infants, and concern has been expressed about the possible harmful effect of such fluctuations in premature neonates. ${ }^{11}$ Most, if not all, the studies used sedation or anaesthesia to ensure that the infant was still throughout the scan. These studies have looked at the evolution of the normal brain and pathology in premature neonates. They have shown the value of MR in these circumstances, especially in the assessment of brain damage resulting from perinatal asphyxia, for which the MR images provide important clues to the timing and nature. ${ }^{6}$ The results will affect not only the clinical management but are likely to provide prognostic information and have medicolegal consequences.

In this study we have shown that low field strength MR has particular advantages over ultrasound in the clinical setting. Its multiplanar ability is a distinct advantage for looking at structural abnormalities such as in fig 4 . Here the ultrasound image showed dilated ventricles and a structural defect on the surface of the brain; the diagnosis of holoprosencephaly was made from the series of MR images. This had important clinical implications as it altered the management and prognosis. This was also the case with the infant in fig 5 who had hydranencephaly. The MR image (fig 5A) showed the absence of the cortical rim of tissue. The ultrasound scan (fig 5B) could not exclude peripheral tissue, as the periphery of the brain is difficult to visualise with ultrasound.

Figure 6 shows the brain of an infant who had a large posterior fossa bleed resulting in respiratory arrest. The MR image (fig 6A) shows the blood situated extra-axially in the posterior fossa. It compresses the cerebellar hemispheres and tracks above the tentorium. It could not be seen on the ultrasound images (fig 6B).

In the three cases of hypoxic-ischaemic injury in this study, the MR images were abnormal when the ultrasound images were initially normal, and only at the later stages when cysts developed did the MR and ultrasound scans have similar appearances (fig 7).

In other clinical cases, the information provided by the two imaging techniques was identical. The infant imaged in fig 8 had an intraventricular cyst that was seen initially on antenatal ultrasound and MR images and followed up with postnatal ultrasound (fig 8B) and MR (fig 8A). 
Table 2 Abnormal cases where magnetic resonance (MR) provided more information than ultrasound or even changed the diagnosis

\begin{tabular}{lll}
\hline MR diagnosis & Ultrasound diagnosis & $\begin{array}{l}\text { Number of } \\
\text { patients }\end{array}$ \\
\hline Small subdural haematoma & Normal & 5 \\
Hypoxic ischaemic changes & Normal & 5 \\
PVL and defined the extent & Possible PVL & 2 \\
Holoprosencephaly & Porencephalic cyst & 1 \\
Leucodystrophy & Normal & 1 \\
Acute middle cerebral artery territory infarct & Normal & 1 \\
Schizencephaly \& hydrocephalus & Hydrocephalus & 1 \\
Lissencephaly \& hydrocephalus & Hydrocephalus & 1 \\
Subarachnoid haemorrhage & Normal & 1 \\
Hydranencephaly & Gross hydrocephalus & 1 \\
Posterior fossa bleed & Normal & 1 \\
Heterotopic grey matter & Normal & 1 \\
Abnormal signal after meningitis (? significance) & Normal & 1 \\
Normal & Nallosal agenesis & 1 \\
Excess CSF space (? significance) & Normal & 1 \\
\hline & &
\end{tabular}

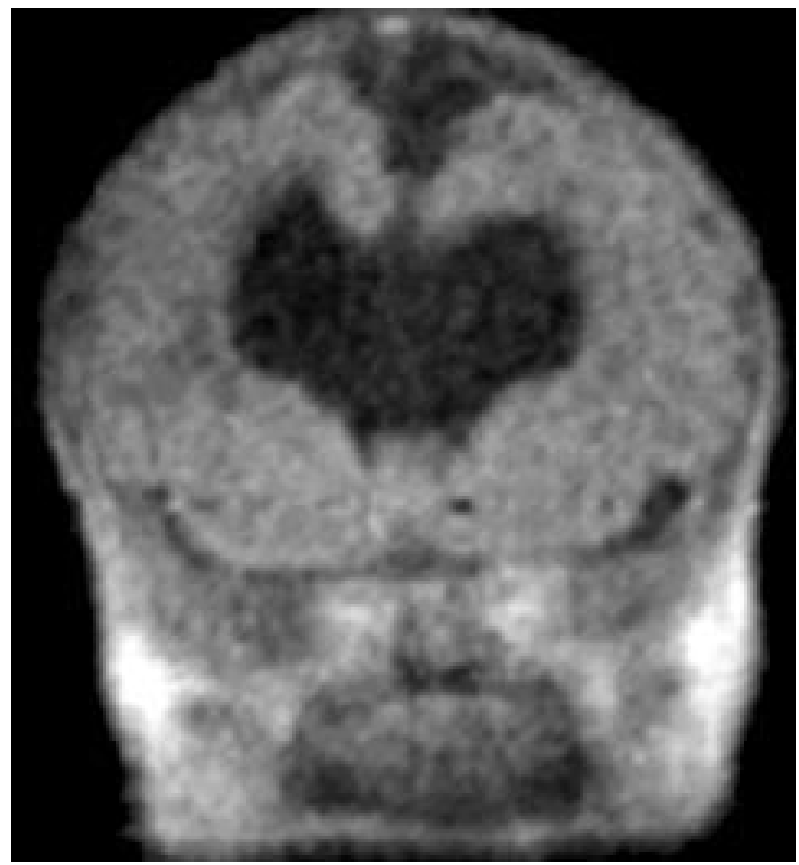

Figure $4 \mathrm{Tl}$ weighted coronal image showing holoprosencephaly. Magnetic resonance showed the brain abnormality clearly over several image slices including other features of holoprosencephaly not seen on this slice.

Low field strength MR offers an imaging protocol that is more effective than ultrasound especially in the posterior fossa and peripheral regions of the brain. Ultrasound appears superior in detecting early and small germinal matrix bleeds.

The low field strength means that the fringe field can be contained within the small RF screening enclosure. The enclosure has two access doors (front and side), and two sides are constructed of RF mesh windows to provide good visibility. It is possible for a member of the medical team to sit inside the enclosure with the baby. Full resuscitation equipment is on site, and at least one of the personnel present at the time of scanning is trained in paediatric resuscitation. The open magnet aperture $(750 \times 200 \mathrm{~mm})$ gives good access to the babies so that they can be mechanically ventilated throughout the scan.

The overall cost for each MR scan is estimated at $£ 60$, similar to the cost of an ultrasound examination. The initial capital costs for the machines are also similar at $£ 150000$.
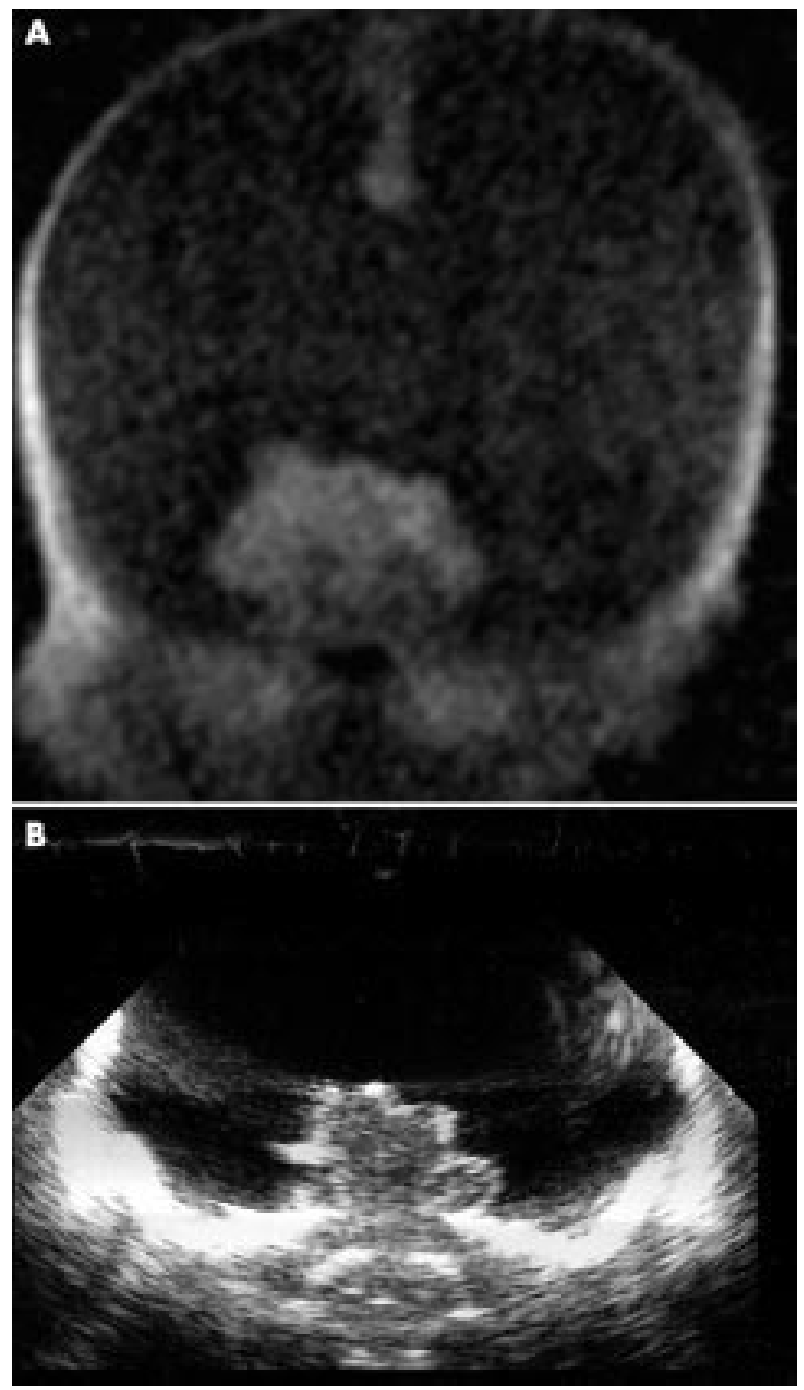

Figure $5 \mathrm{Tl}$ weighted coronal image $(\mathrm{A})$ and ultrasound image (B). Hydranencephaly is seen on both imaging modalities, but was easily detected by magnetic resonance. Massive hydrocephalus could not be completely excluded by ultrasound (although it was less likely).

The ability to site conventional ventilation and resuscitation equipment close to the magnet provides an advantage for the 

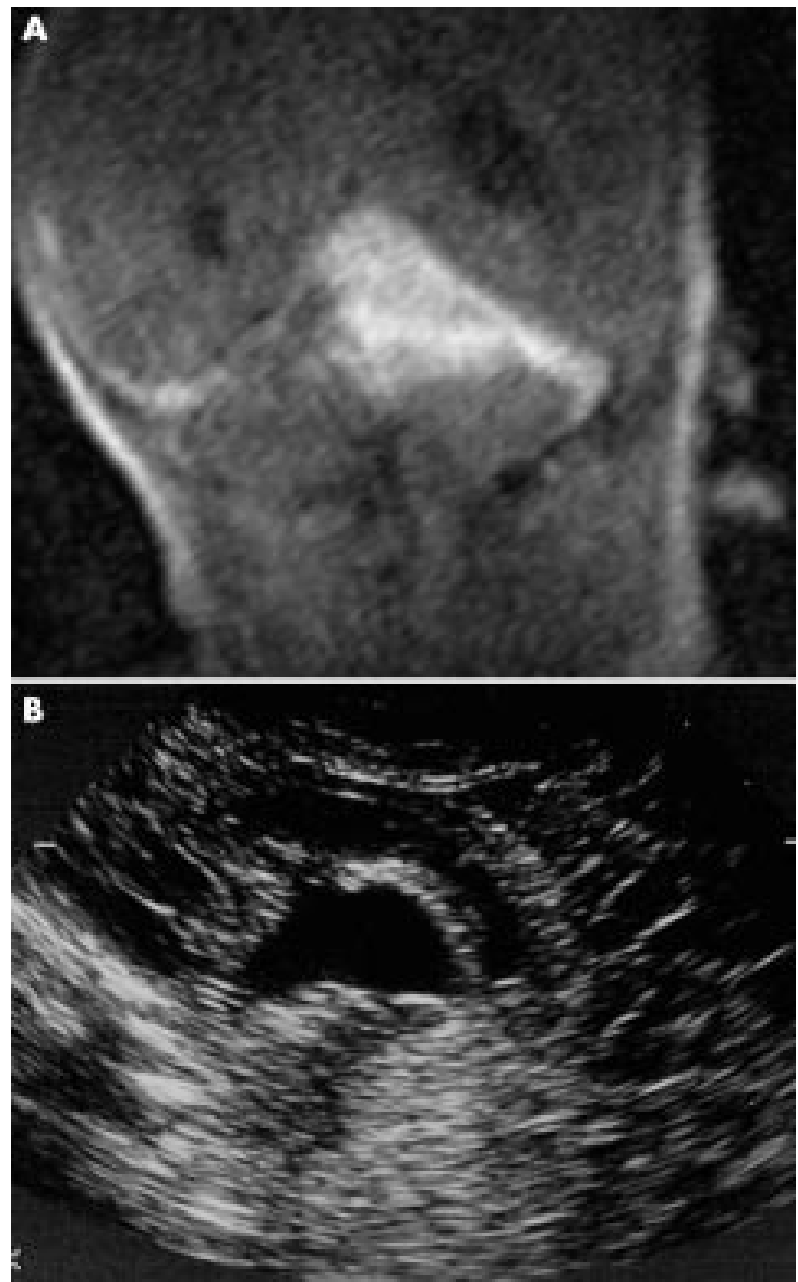

Figure 6 T1 weighted coronal image $(A)$ and ultrasound sagittal image (B). A posterior fossa bleed is clearly seen on the magnetic resonance image but is poorly visualised using ultrasound.

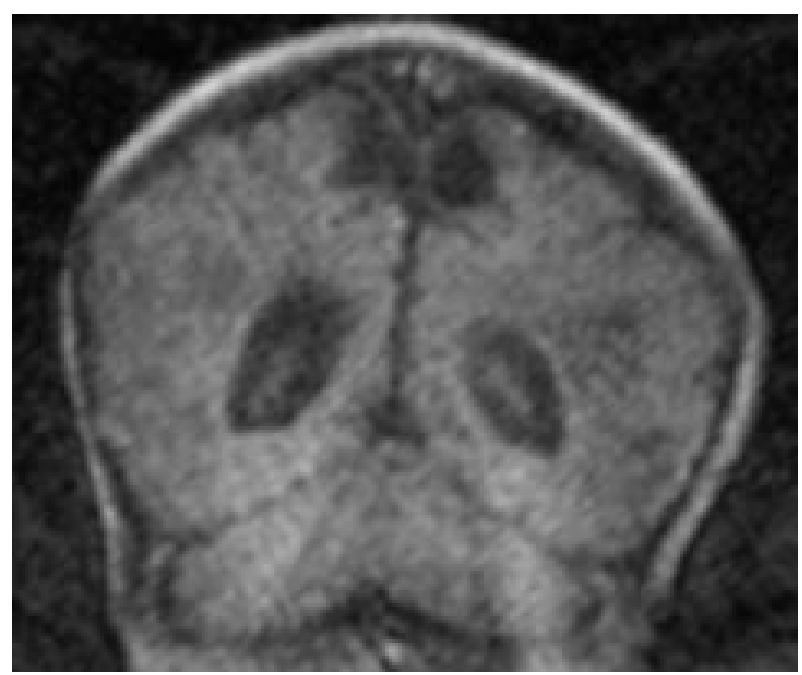

Figure 7 Tl weighted coronal image. Late hypoxic-ischaemic changes can be seen (on both ultrasound and magnetic resonance imaging).

dedicated niche magnet over conventional high field MR systems in which specialised MR compatible equipment must be used.
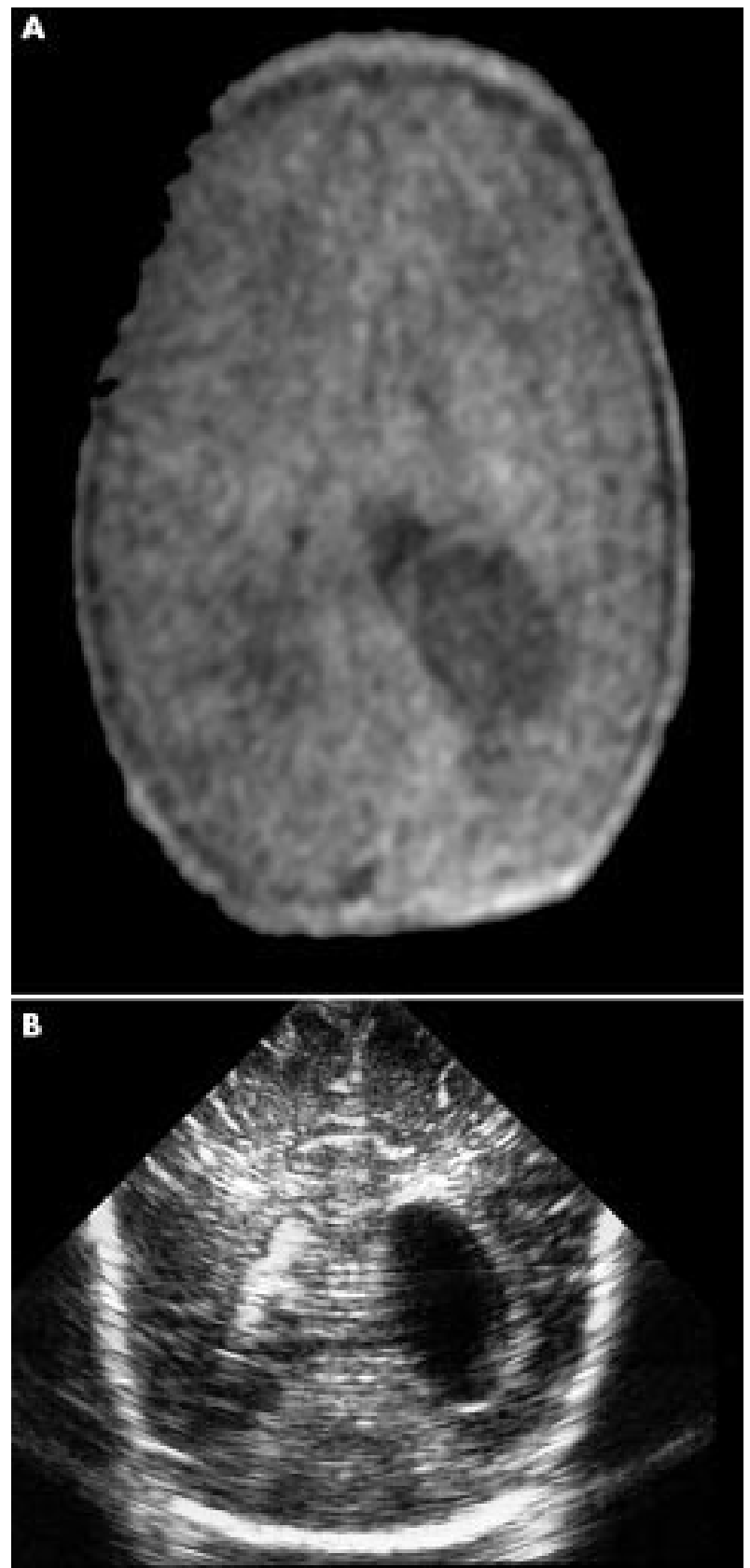

Figure 8 Tl weighted axial magnetic resonance image $(\mathrm{A})$ and ultrasound image (B). An intraventricular cyst can be seen equally well on both images.

At high field, the delicate premature neonate whose thermoregulatory system may not yet be fully developed may be exposed to relatively high radiofrequency absorption, particularly with modern techniques such as fast spin echo. Acoustic noise is also a serious problem when scanning neonates at high field, when sound levels may easily exceed $100 \mathrm{dBA}$. The low field scanner has a very low radiofrequency absorption and is almost inaudible, posing no risk to hearing. No untoward effects on vital signs have been observed in any of our studies when the baby has entered the magnet, or scanning has started, unlike effects reported at high field. ${ }^{11}$ Open physical access means the baby can be rapidly removed in case of adverse events. In this work we have assumed that"experimental" MR is the optimum method as it has detected additional pathology to the ultrasound. However, we do not 
have an independent arbiter such as a post mortem examination, except in one case, in which the post mortem examination agreed with the MR report.

Owing to the low field strength of the magnet, the acquisition time for T2 weighted images is over five minutes resulting in a high risk of movement artefacts. The long echo times required to achieve T2 weighting (TE typically 120-200 milliseconds) in the neonatal brain (T2 typically 1000-1500 milliseconds) mean that signal to noise ratio is low for these scans. The gradient echo sequence for the detection of haemorrhages is not yet optimal, and diffusion weighted imaging for the early detection of ischaemia is also difficult because of motion related problems (real time imaging has not yet been implemented on the dedicated system). These problems are currently being resolved by further development of the scanner. ${ }^{12}$

MR will not replace ultrasound as the routine imaging modality on the neonatal intensive care unit but can provide important additional information in selected cases. It is ideal for all brain malformations that are difficult to fully characterise by ultrasound. In certain cases, such as hypoxic injury, an MR scan can provide information over and above that acquired with ultrasound.

Further research is needed before its value in prognosis is established; all the infants in this study are being followed up.

\section{Conclusion}

Low field strength MR scanning is well tolerated by neonates and provides a valuable additional modality to ultrasound on the neonatal intensive care unit. This study has confirmed the ability to scan neonates with little disruption of their external environment and has also provided a database of normal appearances at different gestation. In this study, babies were shown to have lesions not detected by ultrasound which were clinically significant. MR imaging will not replace ultrasound as the main imaging modality on the neonatal intensive care unit but can provide an important additional imaging method when the ultrasound findings are inconclusive. Dedicated MR scanners have similar capital and operating costs to ultrasound machines and so could be much more widely used, particularly with the increasing availability of web based and teleradiology services to provide specialised MR radiological reporting. The information provided will be important in both patient management and parent counselling.

n...................

Authors' affiliations

E H Whitby, M N Paley, N Woodhouse, P D Griffiths, Section of Academic Radiology, University of Sheffield, Sheffield, UK

M F Smith, Trent Regional Neonatal Unit, Jessop Hospital, Sheffield, UK

A Sprigg, Sheffield Children's Hospital, Sheffield, UK

\section{REFERENCES}

1 Boyer R S. Neuroimaging of premature infants. Imaging clinics of North America. Paediatr Neuroradiol 1994:4:241-61.

2 Cohen HL, Haller JO. Advances in perinatal neurosonography. AJR Am J Roentgenol 1994;163:801-10.

3 O'Shea TM, Volberg F, Dillard RG. Reliablitiy of interpretation of crania ultrasound examinations of very low birth weight neonates. Dev Med Child Neurol 1993;35:97-101.

4 Blankenberg FG, Norbash AM, Lane B, et al. Neonatal intracranial ischaemia and haemorrhage: diagnosis with US,CT, and MR imaging Radiology 1996:199:253-9.

5 McArdle CB, Richardson CJ, Hayden CK, et al. Abnormalities of the neonatal brain: MR imaging. Part 11 . Hypoxic-ischaemic brain injury. Radiology 1987; 163:395-403.

6 Barkovich AJ, Truwit $\mathrm{Cl}$. Brain damage from perinatal asphyxia: correlation of MR findings with gestational age. AJNR Am J Neuroradiol 1990:11:1087-96.

7 Keeney S, Adcock EW, McArdle CB. Prospective observations of 100 high risk neonates by high field (1.5T) magnetic resonance imaging of the central nervous system. II. Lesions associated with hypoxic ischaemic encephalopathy. Pediatrics 1991;87:431-8.

8 Latchaw RE, Truwit CE. Imaging of perinatal hypoxic ischaemic brain injury. Semin Pediatr Neurol 1995;2:72-9.

9 Blickman JG, Jaramillo D, Cleveland RH. Neonatal cranial ultrasonography. Current Problems in Diagnostic Padiology 1991:20:91-119.

10 The National Radiation Protection Board ad hoc advisory group on nuclear magnetic resonance clinical imaging. BrJ Radiol 1983:56:974-7.

11 Taber K, Hayman LA, Northrup R, et al. Vital sign changes in infant magnetic resonance examinations. J Magn Reson Imaging 1998:8:1252-6.

12 Paley MNJ, McGinley J. Specialised MRI systems: design, operation and economics. Developments in Magnetic Resonance 1997; 1:76-83.

\section{Archimedes}

In order to give the best care to patients and families, paediatricians need to integrate the highest quality scientific evidence with clinical expertise and the opinions of the family. Archimedes is a bimonthly section in Archives which seeks to assist practising clinicians by providing "evidence based" answers to common questions which are not at the forefront of research but are at the core of practice.

The format of Archimedes may be familiar. A description of the clinical setting is followed by a structured clinical question and a brief report of the search. The best evidence available to answer the question is provided as a summary table (which is electronically linked to more detailed appraisals). To pull the information together, a commentary follows. Finally, to make it all much more accessible, a box provides the clinical bottom line.

This month the following topics have been published:

- Should preterm neonates with a central venous catheter and coagulase negative staphylococcal bacteraemia be treated without removal of catheter?

- Is gradual introduction of feeding better than immediate normal feeding in children with gastroenteritis?

- Are follow up chest $x$ ray examinations helpful in the management of children recovering from pneumonia?

Previous Archimedes questions can be found in the issues of Archives published in the Fetal and Neonatal edition months since September 2001. Readers wishing to submit their own questions-with best evidence answers-are encouraged to read the Instructions for Authors at http://www.archdischild.com. 